Article

\title{
Novel candidates for palladium alloys - enhanced strength and ductility - generalized stacking fault energy calculations
}

\author{
Marek Muzyk
}

Citation: Muzyk, M.. Novel candidates for palladium alloys - enhanced strength and ductility - generalized stacking fault energy calculations Metals 2021, 11, x. https://doi.org/10.3390/xxxxx
Faculty of Mathematics and Natural Sciences. School of Exact Sciences, Cardinal Stefan Wyszynski University in Warsaw, Woycickiego 1/3, 01-938 Warsaw, Poland; e-mail: m.muzyk@uksw.edu.pl, Tel.: +48 225699670

\begin{abstract}
Generalized stacking fault energies of palladium alloys were calculated using the density functional theory. The stacking fault energy of palladium alloys is correlated with the valence electron of the transition metal element. The twinning tendency is also modified by the presence of an alloying element in the plane of deformation. The obtained results suggest that $\mathrm{Pd}$-transition metal alloys with elements such as $\mathrm{Cr}, \mathrm{Mo}, \mathrm{W}, \mathrm{Mn}$, Re are expected to exhibit high work hardening rate due to the tendency to emit of the partial dislocations and mechanical twins, which results in increased strength and ductility.
\end{abstract}

Keywords: palladium; palladium alloys; generalized stacking fault energy; twinnability; stacking fault energy; ab initio calculations

\section{Introduction}

Noble metals are an interesting group of materials due to their specific properties, i.e. conductivity, chemical and thermal stability, which are widely used in functional applications [1]. Mechanical properties of noble metals have also been recently investigated for stacking fault energy (SFE), partial dislocation emission (PDE) and mechanical twinning (MT) [2-4]. Metals with low SFE exhibit high density of stacking faults (SFs) and twins, which results in high ductility and strength[5]. The MT of metals with high SFE can significantly improve the mechanical properties, but it is observed only in ultrafine materials [6]. However, twinnability can be facilitated by an alloying element that decreases SFE $[7,8]$.

Moreover, Tadmor and Bernstein showed that the deformation twinning tendency in a face-centered cubic (fcc) lattice depends on the unstable stacking fault energy (USFE) and the unstable twinning energy (UTE) [9]. The USFE and the UTE are the maximum energies reached during formation of stacking fault and twin, respectively, which can only be estimated by atomic-scale calculations. Tadmor's and Bernstein's method is based on the estimation of the generalized stacking fault energy (GSFE) for specific alloys. GSFE has been computed for a number of fcc metals and their alloys, including Al [7], $\mathrm{Cu}$ [10], $\mathrm{Ni}$ [11]. Experimental examination confirmed that GSFE calculations predicted correctly the lower SFE and twinning occurrence. Calculations provided that even in the case of $\mathrm{Al}$ $\left(\mathrm{SFE}=162 \mathrm{~mJ} / \mathrm{m}^{2}\right)$, some alloys have promising twinnability properties $[7,12]$, and then the twins were observed in Al alloys by transmission electron microscopy (i.e. in Al-Li [13], Al-Mg [14], Al-Mg-Si [15]).

Palladium exhibits unique chemical and mechanical properties [16]. PDE and MT are not observed in this material when in a coarse grained form due to high SFE [6].

However, Kurmanaeva et al. observed that adding Ag to the Pd matrix leads to formation of SFs and twins; strength and ductility are also improved [17]. Many other elements can have a similar effect on Pd properties as Ag, but systematic GSFE studies of Pd alloys have not been performed yet.

In our paper the GSFE of 23 Pd-transition metal alloys have been calculated. SFE, USFE, UTE and twinning energy (TE) were analyzed in terms of PDE and MT. The most 
promising Pd alloys from the MT point of view were selected. Our calculations predict that Pd alloys containing elements such as $\mathrm{Cr}, \mathrm{Mo}, \mathrm{W}, \mathrm{Mn}$, and Re can have increased strength and ductility than Pd-Ag.

\section{Methods}

The calculations were based on the density functional theory (DFT) implemented in the Vienna Ab-initio Simulation Package (VASP). GSFE calculations were performed using projector augmented wave potentials and generalised gradient approximation, with electron exchange-correlation described by Perdew-Burke-Ernzerhof (PBE) parameterization [18-21]. The electronic occupancy of $0.2 \mathrm{eV}$ Fermi smearing and plane-wave cut-off energy of $400 \mathrm{eV}$ were used. The Monkhorst-Pack scheme was used to sample the Brillouin zone with an 8x8x1 k-point mesh. Ion relaxation was terminated when the energy convergence was greater than $10^{-4} \mathrm{eV}$. GSFEs were calculated using a periodic supercell of twelve (111) planes with a 2x2 slab geometry giving 48 atoms in the system. The energies were calculated for the configurations obtained by displacing one part of the crystal on the remaining part towards $<112$ > on the (111) plane. GSFE was calculated by displacing a part of the model in two operations. In the first process, half of the crystal was displaced (1-6 layers of the supercell). In the second step, the second part of the crystal was shifted in the opposite direction (8-12 layers of the supercell) (see Figure 1 in Ref. [7]). GSFE calculations were performed for $\mathrm{Pd}$ and systems with one $\mathrm{Pd}$ atom in the slip plane replaced with an alloying element. Taking into account the size of the supercell, the concentration of the alloying elements was approximately $2 \%$ at. The vacuum space $(6 \AA)$ and relaxation of the position of atoms perpendicular to the free surface were applied. The shape and volume of the cells have been kept fixed during relaxation.

\section{Results and discussion}

GSFE of Pd and their selected alloys are shown in Figure 1. Each line represents Pd or one Pd alloy deformed on (111) plane along the «112〉 direction. First maximum on the plot is the unstable stacking fault energy (USFE); this is the energy barrier on the path to stacking fault formation. First minimum is stacking fault energy (SFE). Second maximum is the unstable twinning energy (UTE); this is the energy barrier to twin formation. Second minimum is the twin energy (TE) and TE is located at 2.0 displacement unit. The USFE, SFE, UTE and TE values for Pd and Pd alloys are included in the Appendix A in comparison with the available experimental and computational results (see Table A.1 and Table A.2).

The SFE, USFE and UTE are shown in Figure 2 as a function of the valence electrons (VEs) of the alloying element. Palladium SFE is equal $148 \mathrm{~mJ} / \mathrm{m}^{2}$ (i.e. SFE of Cu is $45 \mathrm{~mJ} / \mathrm{m}^{2}$ [10], SFE of $\mathrm{Al}$ is $162 \mathrm{~mJ} / \mathrm{m}^{2}$ [12]). SFE values for $\mathrm{Pd}$ alloys showed characteristic trend with a minimum for 5-6 VEs (3d: V, 4d: Mo, 5d: W). The SFE value increases slightly below the pure Pd value for 9-10 VEs metals ( $3 \mathrm{~d}: \mathrm{Co}, \mathrm{Ni}$; $4 \mathrm{~d}$ : Rh; $5 \mathrm{~d}$ : $\mathrm{Ir}, \mathrm{Pt}$ ) and decreases for the filled d-band elements $(\mathrm{Cu}, \mathrm{Ag}, \mathrm{Au})$.

USFE (Figure $2 b$ ) exhibits higher values for almost all the alloying elements considered, except $\mathrm{Ag}$ and $\mathrm{Au}$. 3d (Ti-Cu) alloying elements change the USFE slightly. Elements $4 \mathrm{~d}$ and $5 \mathrm{~d}$ follow a very similar trend, namely increasing to $8 \mathrm{VEs}(\mathrm{Ru}, \mathrm{Os})$ and then decreasing below the value of pure Pd $\left(215 \mathrm{~mJ} / \mathrm{m}^{2}\right)$ for $\mathrm{Ag}$ and $\mathrm{Au}\left(196 \mathrm{~mJ} / \mathrm{m}^{2}\right.$ and $200 \mathrm{~mJ} / \mathrm{m}^{2}$, respectively). 


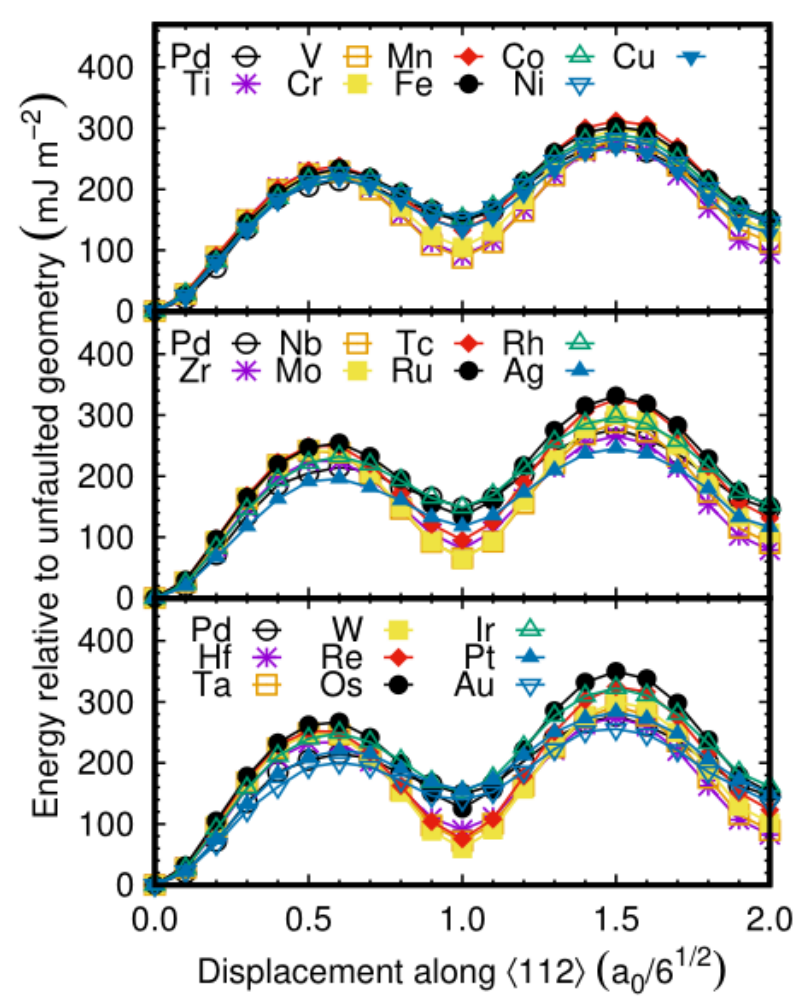

Figure 1. The generalised stacking fault energy (GSFE) of Pd and Pd-transition metal alloys. 3d-, $4 \mathrm{~d}-$ and $5 \mathrm{~d}$-shell electron elements are presented from top to bottom.

The UTE value trend reaches its maximum for: 7 VEs in $3 \mathrm{~d} ; 8$ VEs in $4 \mathrm{~d}$ and $5 \mathrm{~d}$, respectively. As shown in Figure 2c), the UTE values change similarly for $3 \mathrm{~d}, 4 \mathrm{~d}$ and $5 \mathrm{~d}$ alloying metals as a VEs function, except for 8-9 VEs metals, where the difference is visible. The similar trend has been observed in Ni-transition metals alloys. The maximum of SFE is located for 10 VEs metals as for Pd alloys. The minimum of SFE in Ni alloys is almost located at the same position (3d: V, 4d: Mo, 5d: Re) [22,23].

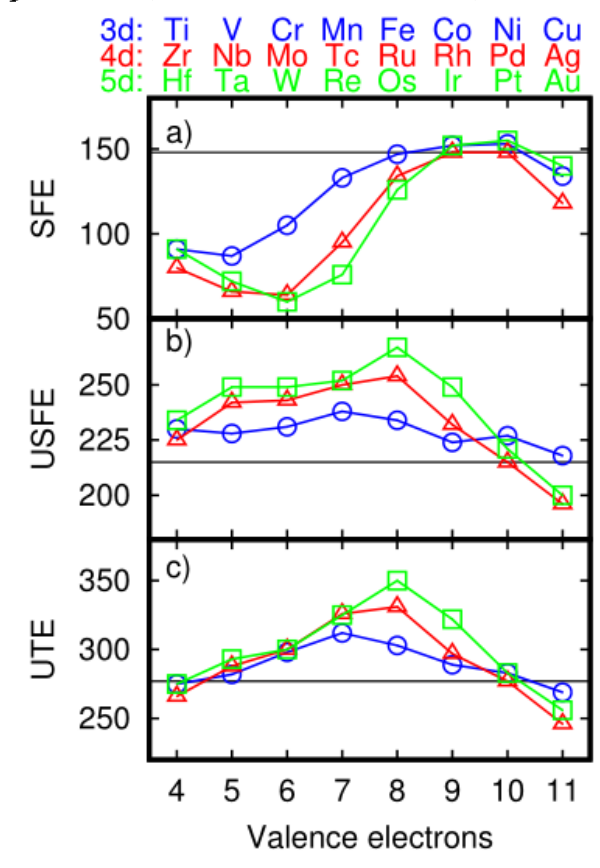

Figure 2. Energy trends: a) stacking fault energy (SFE), b) unstable stacking fault energy (USFE), c) unstable twinning energy (UTE) in Pd transition metals alloys as a function of valence electrons (VEs) in the alloy element. Black tiny lines are the analyzed Pd value (SFE $=148 \mathrm{~mJ} / \mathrm{m}^{2}, \mathrm{USFE}=215$ $\mathrm{mJ} / \mathrm{m}^{2}, \mathrm{UTE}=277 \mathrm{~mJ} / \mathrm{m}^{2}$ ). SFE has a lower value for 5 VEs metals in period $3 \mathrm{~d} ; 6 \mathrm{VEs}$ in periods $4 \mathrm{~d}$ 
and $5 \mathrm{~d}$. USFE and UTE both reach maximum at 8 VEs, then the energies drop. All energies are in $\mathrm{mJ} / \mathrm{m}^{2}$.

The deformation mechanism and twinning tendency are expected to depend on the values of SFE/USFE and UTE/USFE [9], which was confirmed [12,24]. The criteria have been developed by Rice [25] and Tadmor et al [26] based on the concept of Peierls. Analysis of the nucleation of dislocation from the crack tip leads to understanding that depth of SF and TE compared to USFE and UTE is crucial for the preferred deformation mechanism, respectively. When the analyzed alloying element causes these parameters to be lower than in pure metal (a comparable state), the PDE and MT are facilitated [10]. As can be seen from SFE/USFE ratio, the formation of SF is facilitated by alloying elements, which decrease the SFE and increase the USFE compared to Pd (the reference value of pure Pd is marked by black tiny lines in Figure 2 and Figure 3). From Figure 2a and Figure $2 \mathrm{~b}$, we see that these elements are at the begining of each period. Figure 3 illustrates the ratio of SFE/USFE and UTE/USFE as a function of VEs. The curvature of the SFE/USFE ratio is similar to that of the SFE (see Figure 2a). The minium is located for 5 VEs (V) and 6 VEs (Mo, W), then the SFE/USFE ratio increases to a value close to Pd for $10 \mathrm{VEs}(\mathrm{Ni}$, $\mathrm{Pt})$. In the case of metals with a full d-electron shell $(\mathrm{Cu}, \mathrm{Ag}, \mathrm{Au})$ the value drops again. Note that several elements bring the SFE/USFE even below 0.3 ( Nb, Ta, Mo, W, Re). To the best of the authors' knowledge, these alloys have not been tested yet for the deformation mechanism. However with V-Pd alloys, SFs during deformation are widely observed [27]. This is an important experimental study that supports the results of our calculations. Pd with added V has the lowest SFE in all $3 \mathrm{~d}$ transition metal impurities. The calculated SFE/USFE value of the Pd-V alloys is 0.383 . SF emission should be easier with alloying metals with a lower SFE/USFE ratio than with Pd-V alloy (i.e. Pd-Mo, Pd-W).

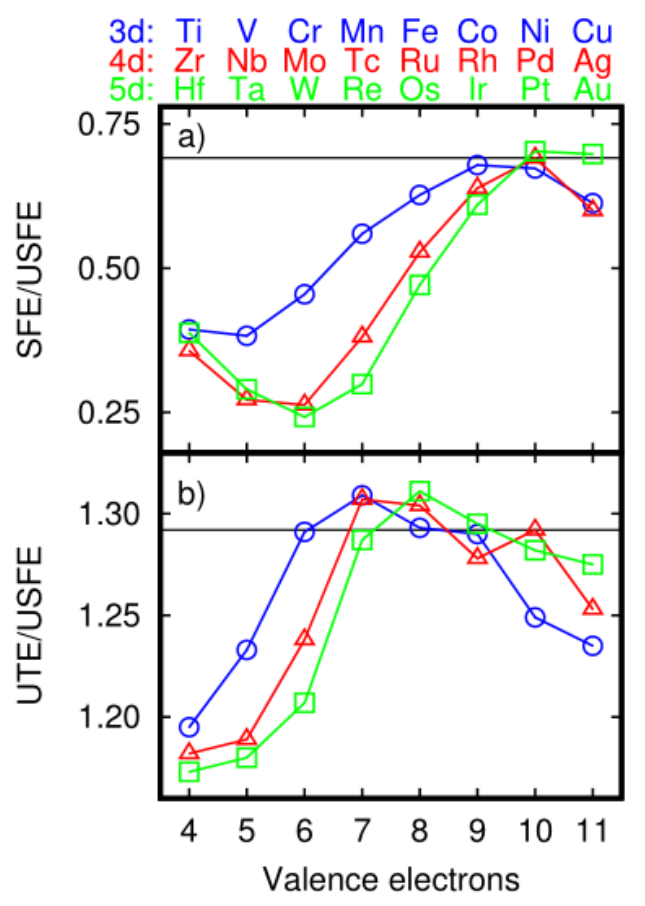

Figure 3. a) Ratio of the stacking fault energy (SFE) to the unstable stacking fault energy (USFE) in Pd transition metal alloys. The thin black line represents the SFE/USFE value for Pd (0.691). The lowest SFE/USFE value means that the alloying element facilitates the partial dislocation emission (PDE). The analyzed value has a specific shape. Zr and Hf greatly increase the value and inhibit PDE. The lowest is for metals with seven valence electrons (VEs), which are the most promising transition metals elements from the PDE point of view. As VEs increases, SFE/USFE increases to the Pd value. b) A measure of twinnability is the ratio of the unstable twinning energy (UTE) to the unstable stacking fault energy (USFE). Increasing UTE/USFE reduces twinning. The tiny black line is the UTE/USFE value for Pd (1.292). The results show a significant trend in twinnability, similar to PDE. 
The lower UTE/USFE ratio increases the twinnability. The UTE/USFE ratio for Pd alloys is equal 1.292 (the value is marked with the black line in Figure 3b). As can be seen in Figure 3b, the deformation twinning is facilitaed by impurity elements with 4-6 VEs (except $\mathrm{Cr}$ ); $11 \mathrm{VEs}$ and $\mathrm{Ni}$ (10 VEs). The remaining elements have no significant influence on the twinnability of $\mathrm{Pd}$, as the UTE/USFE ratio is close to pure palladium ratio. Compared to the SFE/USFE ratio, we can see that elements with 7-8 VEs improve SF formation. By combining the SFE/USFE and UTE/USFE ratios, limited number of impurities can be selected as an interesting candidate from the point of the effect of MT on improving strength and ductility.

Measurements of the twinnability of the Pd alloys proposed in our work confirm the results of experimental tests of Pd-Ag alloys. The addition of silver to the Pd matrix leads a reduction of the average grain size from $240 \mathrm{~nm}$ in pure Pd to $144 \mathrm{~nm}$ in $\mathrm{Pd}-40 \% \mathrm{Ag}$ alloy processed by high pressure torsion; strength and ductility of Pd-Ag alloys improved by $60 \%$ and almost $200 \%$, respectively. The addition of Ag leads to enhanced dislocation storage and strain hardening capacity due to lower stacking fault energy [17]. Calculations also reveal that Ag improves PDE and MT. The computational results of SFE/USFE and UTE/USFE ratio of the Pd-Ag alloy have a beneficial tendency to promote ductility and strength, which is confirmed by experimental studies.

From the group of Pd alloys, only the Pd-Ag alloy has been investigated for strength and ductility. For this reason, we cannot compare our results with any other alloys. On the other hand, Ti-Pd alloys are widely studied due to their high martensitic transformation temperature (MTT) [28]. The increase of the Ti concentration in Pd reduces the MTT which may be due to the facilitation of crystal plane slip. However, the martensitic transformation occurs between different structures to fcc, but the influence of impurities on material properties is generally independent of the crystal structure. The SFE/USFE and UTE/USFE values of the Pd-Ti alloys reveal that Ti significantly improves the Pd plane slip in the fcc structure, which is confirmed by the available experimental studies.

Other results indicate that the addition of copper to the Pd matrix causes a decrease in SFE [29], which is in agreement with our calculations. Unfortunately, there is no information about the mechanical properties of the Pd-Cu alloys.

Similar calculations have been performed for $\mathrm{Cu}$, where thirty $\mathrm{Cu}$ alloys were examined from the point of view of SF and twins formations [10]. The Cu-GSFE calculations showed that 6-7 VEs TMs elements have a significant effect on the SFE/USFE ratio, but twinnability is only slightly modified by impurity. Pd has a potentially greater ability to control the PDE by adding an alloying element, comparing to $\mathrm{Cu}$.

\section{Conclusions}

Pd alloys have not been extensively studied for deformation mechanism. Only the Pd-Ag alloy has been investigated for ductility and strength to the best of the author's knowledge. Our research confirmed that the GSFE calculations provide important materials' properties such as USFE, UTE, which are not available for experimental measurements.

The main results of the GSFE calculations for Pd alloys are summarized in the following points:

- based on GSFE calculations and available experimental studies on Pd transition metal alloys, several promising candidates for alloys with improved strength and ductility were selected;

- the most promising elements in terms of PDE and MT are those with 5-6 VEs;

- $\operatorname{Pd}-\mathrm{X}(\mathrm{X}=\mathrm{Cr}, \mathrm{Mo}, \mathrm{W}, \mathrm{Mn}, \mathrm{Re})$ binary alloys can exhibit extraordinary enhancement of PDE and MT;

- experimental results of Pd-Ag alloys confirmed our GSFE findings that Ag decreased SFE/USFE and UTE/USFE resulting in improved MT and strength;

- based on Pd-Ag alloys, selected Pd alloys may exhibit requested mechanical properties. 
Based on GSFE calculations compared with available experimental results, a new class of Pd alloys was proposed. Experimental verification may deliver interesting insight to Pd alloys properties.

Funding: This research received no external funding.

Acknowledgments: Part of calculations were performed on supercomputers of University of Warsaw in the Interdisciplinary Centre for Mathematical and Computational Modeling (ICM) (Grant no. G63-12). The author would like to thank Elżbieta Kurpiewska for cross-checking calculations using the Quantum Espresso code under her Master thesis.

Conflicts of Interest: The author declares no conflict of interest.

\section{Appendix A}

Table A.1. Calculated values of unstable stacking fault energy (USFE), stacking fault energy (SFE), unstable twinning energy (UTE) and twinning energy (TE) for Pd. The available results of experimental research (Exp.) and other calculations (Calc.) are presented. All energies are in $\mathrm{mJ} / \mathrm{m} 2$.

\begin{tabular}{ccccccc}
\hline & USFE & SFE & UTE & TE & SFE/USFE & USFE/UTE \\
\hline $\mathrm{Pd}$ & 215 & 148 & 277 & 146 & 0.691 & 1.288 \\
\hline & $202[30]$ & $134[30]$ & $261[30]$ & $63[30]$ & $0.663[30]$ & $1.292[30]$ \\
Calc. & $260[29]$ & $143[29]$ & $361[31]$ & $97[34]$ & $0.550[29]$ & $1.259[31]$ \\
& $287[31]$ & $168[31]$ & & & $0.585[31]$ & $0.588[32]$ \\
& $374[32]$ & $220[32]$ & & & \\
& & $225[33]$ & & & \\
Exp. & $180[35,36]$ & & & \\
& & $179[17]$ & & & \\
& $96[2]$ & & & \\
\end{tabular}

Table A.2. Calculated values of unstable stacking fault energy (USFE), stacking fault energy (SFE) and unstable twinning energy (UTE) for Pd and Pd-transition metal alloys. The available results of experimental research and other calculations are presented. All energies are in $\mathrm{mJ} / \mathrm{m} 2$.

\begin{tabular}{|c|c|c|c|c|c|c|c|c|}
\hline 3d: & $\mathrm{Ti}$ & $\mathrm{V}$ & $\mathrm{Cr}$ & Mn & $\mathrm{Fe}$ & Co & $\mathrm{Ni}$ & $\mathrm{Cu}$ \\
\hline USFE & 230 & 228 & 231 & 238 & 234 & 224 & 227 & 218 \\
\hline SFE & 91 & 87 & 105 & 133 & 147 & 152 & 153 & $\begin{array}{c}134 \\
\sim 150[36]\end{array}$ \\
\hline UT & 275 & 282 & 298 & 312 & 303 & 289 & 283 & 269 \\
\hline $\mathrm{TE}$ & 94 & 113 & 131 & 147 & 152 & 151 & 146 & 127 \\
\hline SFE/USFE & 0.394 & 0.383 & 0.455 & 0.560 & 0.627 & 0.679 & 0.673 & 0.613 \\
\hline UTE/USFE & 1.195 & 1.233 & 1.291 & 1.309 & 1.293 & 1.290 & 1.248 & 1.235 \\
\hline $4 \mathrm{~d}:$ & $\mathrm{Zr}$ & $\mathrm{Nb}$ & Mo & Tc & $\mathrm{Ru}$ & $\mathrm{Rh}$ & $\mathrm{Pd}$ & $\mathrm{Ag}$ \\
\hline USFE & 225 & 242 & 243 & 250 & 254 & 232 & 215 & 196 \\
\hline SFE & 80 & 66 & 64 & 95 & 134 & 149 & 148 & $\begin{array}{c}118 \\
81[2] \\
118[17] \\
\sim 120[36] \\
125[38]\end{array}$ \\
\hline UTE & 266 & 288 & 300 & 326 & 331 & 297 & 277 & 246 \\
\hline $\mathrm{TE}$ & 79 & 90 & 107 & 131 & 151 & 153 & 146 & 116 \\
\hline SFE/USFE & 0.357 & 0.272 & 0.263 & 0.381 & 0.528 & 0.639 & 0.691 & 0.600 \\
\hline UTE/USFE & 1.182 & 1.189 & 1.238 & 1.307 & 1.304 & 1.278 & 1.292 & 1.253 \\
\hline
\end{tabular}




\begin{tabular}{|c|c|c|c|c|c|c|c|c|}
\hline 5d: & $\mathrm{Hf}$ & $\mathrm{Ta}$ & $\mathrm{W}$ & $\operatorname{Re}$ & Os & $\mathrm{Ir}$ & $\mathrm{Pt}$ & $\mathrm{Au}$ \\
\hline USFE & 234 & 249 & 249 & 252 & 267 & 249 & 221 & $\begin{array}{c}200 \\
\sim 210[4]\end{array}$ \\
\hline SFE & 91 & 72 & 60 & 76 & 126 & 152 & 155 & $\begin{array}{c}140 \\
\sim 150[4]\end{array}$ \\
\hline UT & 275 & 293 & 300 & 325 & 350 & 322 & 283 & $\begin{array}{c}256 \\
\sim 280[4]\end{array}$ \\
\hline $\mathrm{TE}$ & 83 & 90 & 102 & 123 & 152 & 161 & 149 & 135 \\
\hline SFE/USFE & 0.388 & 0.290 & 0.242 & 0.299 & 0.471 & 0.610 & 0.703 & 0.698 \\
\hline UTE/USFE & 1.173 & 1.180 & 1.207 & 1.287 & 1.311 & 1.295 & 1.282 & 1.275 \\
\hline
\end{tabular}

\section{References}

1. Yan, Y.; Wang, T.; Li, X.; Pang, H.; Xue, H. Noble metal-based materials in high-performance supercapacitors. Inorg. Chem. Front. 2017, 4, 33, doi:10.1039/c6qi00199h.

2. Rao, P.R.; Rao, K.K.; Rao, K.K. X-Ray Measurements of Faulting in Ag-Pd Alloys. J. Appl. Phys. 1968, 39, 4563, doi:10.1063/1.1655801.

3. Leibner, A.; Braun, C.; Heppe, J.; Grewer, M.; Birringer, R. Plastic yielding in nanocrystalline Pd-Au alloys mimics universal behavior of metallic glasses. Phys. Rev. B. 2015, 91, 174110, doi:10.1103/PhysRevB.91.174110.

4. Schafer, J.; Stukowski, A.; Albe, K. Plastic deformation of nanocrystalline Pd - Au alloys: On the interplay of grain boundary solute segregation, fault energies and grain size. Acta Mater. 2011, 59, 2957-2968, doi:10.1016/j.actamat.2011.01.036.

5. Lu, L.; Chen, X.; Huang, X.; Lu, K. Revealing the Maximum Strength in Nanotwinned Copper. Science (80-. ). 2009, 323, 607610, doi:10.1126/science.1167641.

6. Ivanisenko, Y.; Fecht, H. Mechanical behavior of nanocrystalline palladium at high strains and high strain rates. Mater. Sci. Forum. 2008, 584-586, 203-208, doi:10.4028/www.scientific.net/MSF.584-586.203.

7. Muzyk, M.; Pakiela, Z.; Kurzydlowski, K.J. Ab initio calculations of the generalized stacking fault energy in aluminium alloys. Scr. Mater. 2011, 64, 916-918, doi:10.1016/j.scriptamat.2011.01.034.

8. Cai, T.; Zhang, Z.J.; Zhang, P.; Yang, J.B.; Zhang, Z.F. Competition between slip and twinning in face-centered cubic metals. J. Appl. Phys. 2014, 116, 163512, doi:10.1063/1.4898319.

9. Tadmor, E.B.; Bernstein, N. A first-principles measure for the twinnability of FCC metals. J. Mech. Phys. Solids. 2004, 52, 25072519, doi:10.1016/j.jmps.2004.05.002.

10. Muzyk, M.; Kurzydłowski, K.J. Generalised Stacking fault energies of copper alloys - density functional theory calculations. J. Min. Metall. Sect. B Metall. 2019, 55, 271-282, doi:10.2298/JMMB181128020M.

11. Siegel, D.J. Generalized stacking fault energies, ductilities, and twinnabilities of Ni and selected Ni alloys. Appl. Phys. Lett. 2005, 87, 121901-121903, doi:10.1063/1.2051793.

12. Muzyk, M.; Pakieła, Z.; Kurzydłowski, K.J. Generalized Stacking Fault Energies of Aluminum Alloys-Density Functional Theory Calculations. Metals (Basel). 2018, 8, 823, doi:10.3390/met8100823.

13. Ahmad, S.I.; Al-sulaiti, L.A.; Mkhoyan, K.A.; Youssef, K.M. Artifact-free bulk nanocrystalline Al-Li alloys with multiple deformation mechanisms and improved tensile properties. Mater. Today Commun. 2020, 25, 101607, doi:10.1016/j.mtcomm.2020.101607.

14. Velasco, L.; Hodge, A.M. Growth twins in high stacking fault energy metals : Microstructure, texture and twinning. Mater. Sci. Eng. A. 2017, 687, 93-98, doi:10.1016/j.msea.2017.01.065.

15. Liu, M.; Roven, H.J.; Yu, Y.; Werenskiold, J.C. Deformation structures in 6082 aluminium alloy after severe plastic deformation by equal-channel angular pressing. Mater. Sci. Eng. A. 2008, 483-484, 59-63, doi:10.1016/j.msea.2006.09.144.

16. Edalati, K.; Akama, D.; Nishio, A.; Lee, S.; Yonenaga, Y.; Cubero-Sesin, J.M.; Horita, Z. Influence of dislocation - solute atom interactions and stacking fault energy on grain size of single-phase alloys after severe plastic deformation using high- 
pressure torsion. Acta Mater. 2014, 69, 68-77, doi:10.1016/j.actamat.2014.01.036.

17. Kurmanaeva, L.; Ivanisenko, Y.; Markmann, J.; Kübel, C.; Chuvilin, A.; Doyle, S.; Valiev, R.Z.; Fecht, H.-J. Grain refinement and mechanical properties in ultrafine grained Pd and Pd - Ag alloys produced by HPT. Mater. Sci. Eng. A. 2010, 527, 17761783, doi:10.1016/j.msea.2009.11.001.

18. Blöchl, P.E. Projector augmented-wave method. Phys. Rev. B. 1994, 50, 17953-17979, doi:10.1103/PhysRevB.50.17953.

19. Perdew, J.P.; Burke, K.; Ernzerhof, M. Generalized gradient approximation made simple. Phys. Rev. Lett. 1996, 77, 3865-3868, doi:10.1103/PhysRevLett.77.3865.

20. Perdew, J.P.; Burke, K.; Ernzerhof, M. Generalized Gradient Approximation Made Simple- ERRATA. Phys. Rev. Lett. 1996, 77, 3865-3868, doi:10.1103/PhysRevLett.77.3865.

21. Joubert, D. From ultrasoft pseudopotentials to the projector augmented-wave method. Phys. Rev. B - Condens. Matter Mater. Phys. 1999, 59, 1758-1775, doi:10.1103/PhysRevB.59.1758.

22. Schang, S.L.; Zacher, C.L.; Fang, H.Z.; Wang, Y.; Du, Y.; Liu, Z.K. Effects of alloying element and temperature on the stacking fault energies of dilute Ni-base superalloys. J. Phys. Condens. Matter. 2012, 24, 505403, doi:10.1088/0953-8984/24/50/505403.

23. Shang, S.; Wang, Y.; Du, Y.; Tschopp, M.A.; Liu, Z. Integrating computational modeling and first-principles calculations to predict stacking fault energy of dilute multicomponent Ni-base alloys. Comput. Mater. Sci. 2014, 91, 50-55, doi:10.1016/j.commatsci.2014.04.040.

24. Brandl, C.; Derlet, P.M.; Van Swygenhoven, H. General-stacking-fault energies in highly strained metallic environments: Ab initio calculations. Phys. Rev. B - Condens. Matter Mater. Phys. 2007, 76, 1-8, doi:10.1103/PhysRevB.76.054124.

25. Rice, J.R. Dislocation nucleation from a crack tip: an analysis based on the Peierls concept. J. Mech. Phys. Solids. 1992, 40, 239271, doi:10.1016/S0022-5096(05)80012-2.

26. Tadmor, E.B.; Hai, S. A Peierls criterion for the onset of deformation twinning at a crack tip. J. Mech. Phys. Solids. 2003, 51, 765-793, doi:10.1016/S0022-5096(03)00005-X.

27. Ghosh, J.; Chattopadhyay, S.K.; Meikap, A.K.; Chatterjee, S.K.; Chatterjee, P. Study of microstructure in vanadium palladium alloys by X-ray diffraction technique. Bull. Mater. Sci. 2007, 30, 447-454, doi:10.1007/s12034-007-0071-0.

28. Yamabe-Mitarai, Y. TiPd- and TiPt-Based High-Temperature Shape Memory Alloys : A Review on Recent Advances. Metals (Basel). 2020, 10, 1531, doi:10.3390/met10111531.

29. MacLaren, J.M. Electronic Structure of Stacking Faults in Pd1-xCux, Pd1-xAgx, and Ag1-xAux Alloys. In: Stability of Materials. Boston,MA.: Springer; 1996:407-412. doi:10.1007/978-1-4613-0385-5_32.

30. Jin, Z.H.; Dunham, S.T.; Gleiter, H.; Hahn, H.; Gumbsch, P. A universal scaling of planar fault energy barriers in face-centered cubic metals. Scr. Mater. 2011, 64, 605-608, doi:10.1016/j.scriptamat.2010.11.033.

31. Kibey, S.; Liu, J.B.; Johnson, D.D.; Sehitoglu, H. Predicting twinning stress in fcc metals: Linking twin-energy pathways to twin nucleation. Acta Mater. 2007, 55, 6843-6851, doi:10.1016/j.actamat.2007.08.042.

32. Cai, J.; Wang, F.; Lu, C.; Wang, Y.Y. Structure and stacking-fault energy in metals Al, Pd, Pt, Ir, and Rh. Phys. Rev. B. 2004, 69, 224104, doi:10.1103/PhysRevB.69.224104.

33. Rosengaard, N.M.; Skriver, H.L. Calculated stacking-fault energies of elemental metals. Phys. Rev. B. 1993, 47, 12865-12873, doi:10.1103/PhysRevB.47.12865.

34. Xu, J.; Lin, W.; Freeman, A.J. Twin-boundary and stacking-fault energies in Al and Pd. Phys. Rev. B. 1991, 43, 2018-2024, doi:10.1103/PhysRevB.43.2018.

35. Hirth, J.P.; Lothe, J. Theory of Dislocations. 2nd ed. Wiley, New York; 1982.

36. Harris, I.R.; Dillamore, I.L.; Smallman, R.E.; Beeston, B.E.P. The influence of d-band structure on stacking-fault energy. Philos. Mag. 1966, 14, 325-333, doi:10.1080/14786436608219015.

37. Lu, S.; Hu, Q.; Delczeg-Czirjak, E.K.; Johansson, B.; Vitos, L. Determining the minimum grain size in severe plastic deformation process via first-principles calculations. Acta Mater. 2012, 60, 4506-4513, doi:10.1016/j.actamat.2012.04.024. 
38. Yang, K.; Ivanisenko, Y.; Caron, A.; Chuvilin, A.; Kurmanaeva, L. Mechanical behaviour and in situ observation of shear bands in ultrafine grained Pd and Pd - Ag alloys. Acta Mater. 2010, 58, 967-978, doi:10.1016/j.actamat.2009.10.013. 